\title{
219. FM Sensitive Auditory Neuron
}

\author{
By Takeshi WatanaBE*) and Kengo OHgushI**) \\ (Comm. by Yas Kuno, M. J. A., Nov. 12, 1968)
}

A number of electrophysiological studies on the activity of single auditory neurons has been reported. Most of these have employed rather simple sound stimuli, such as pure tones and clicks. Hearing is an acoustic cue that analyzes the complex sound. From this viewpoint, the purpose of the present report is to determine the response characteristics of auditory neurons from the periphery to the cortex using frequency-modulated (FM) sound and to investigate their behavioral correlates. Recently, responses of auditory neurons to FII sound have been studied, ${ }^{1)-5}$ ) and the directional sensitivity of the neurons in the upper levels of brain has been recognized. Three types of auditory neurons that respond to FM sound were found in the inferior colliculus of the cat. They were classified according to their sensitivity to ascending (increasing) or descending (decreasing) FM sound and to different rates of frequency modulation. These findings may well explain the neural analysis of FM components in the cat's voice. Also, FM sensitive neurons may play an important role in analyzing complex sounds, such as speech.

Using both glass capillary and tungsten microelectrodes, responses of auditory neurons to FM sound were investigated. FM sound was produced by either a Frequency-Response Tracer (Kokuyo Co.) or a Wavetek oscillator modulated by sinusoid and sawtooth waves. An electric counter was used to monitor the frequency of stimulating sounds. Most of the recordings were stored on magnetic tapes, and subsequent off-line computation was accomplished by the use of a CAT computer. Auditory neurons in the lower levels of brain, that is, the cochlear nerve and the neurons in the cochlear nucleus, did not show any directional sensitivity to FM sound sweeping both within and across of the response area of a neuron. Directional sensitivity of the neurons to FM sound was found at the level of the superior olivary complex where most neurons (66\%) showed greater sensitivity to an ascending rather than descending frequency changes. Conversely, some units $(18 \%)$ were more sensitive to a descending frequency, while others were non-directional in their responses. The neurons in the inferior colliculus, medial geniculate

*) Dept. of Physiology, Tokyo Medical and Dental University, Tokyo.

**) Broadcasting Science Research Laboratories, NHK, Tokyo. 
and the auditory cortex (AI) exhibited distinct directional sensitivity to ascending or descending FM sound. However, some units in the medial geniculate responded only to descending FM sound and did not respond to pure tones at all which, so far, examined the entire auditory frequency range.

The following describes the response pattern of the collicular neurons to FM sound. Fig. 1 shows the unit responses of a neuron to

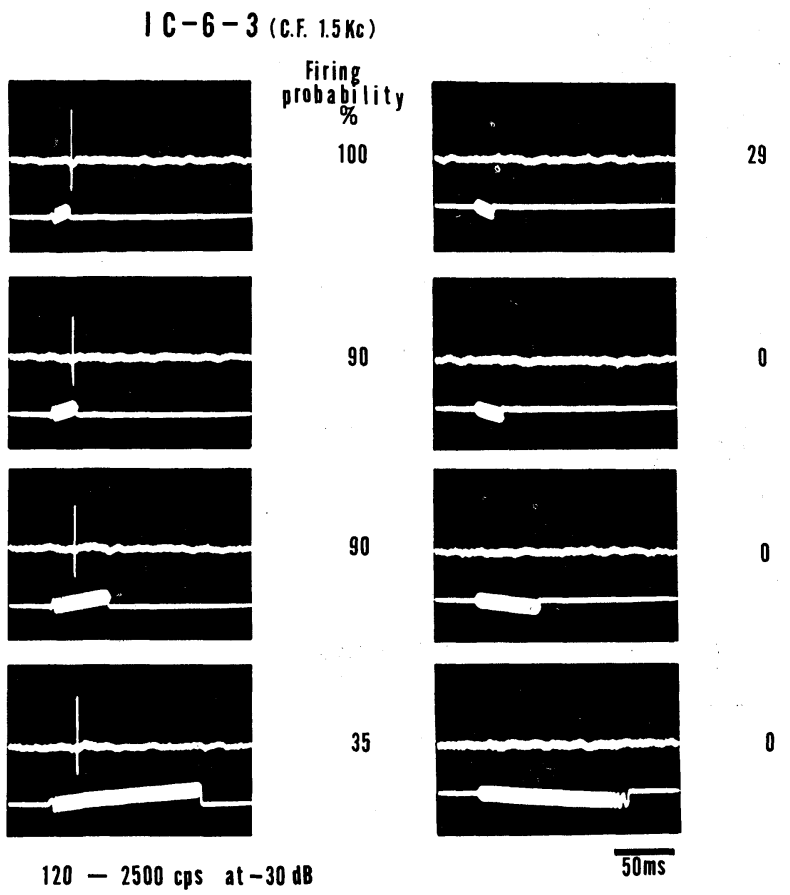

Fig. 1. An ascending FM responding collicular neuron sensitive to a fast rate of modulation. Left and right columns represent an ascending and descending FM series, respectively, at four different rates of modulation. The characteristic frequency of this neuron was $1.5 \mathrm{Kc}$ and the modulating frequency range was between 120 cps and $2500 \mathrm{cps}$. The firing probability is shown at the right of each sample record. In each record, the top trace is the neuronal response and the bottom trace represents the FM stimulus combined with the modulating sawtooth wave with its modulated FM tones.

ascending (left column) and descending (right column) FM sounds at four different rates of modulation. The rate of modulation was altered by changing the duration of FM tones, while the frequency range was held constant between $120 \mathrm{cps}$ and $2500 \mathrm{cps}$. The number shown at the right of each sample record indicates the firing probability of this neuron. It is obvious that this particular unit re- 
sponded mostly to ascending rather than descending FM sound, and that it was more responsive to a fast rate of modulation. An example of neurons found in the inferior colliculus exhibiting descending directional sensitivity to a fast rate of modulation is presented in Fig. 2. Neuronal responses to ascending (left records) and descend-
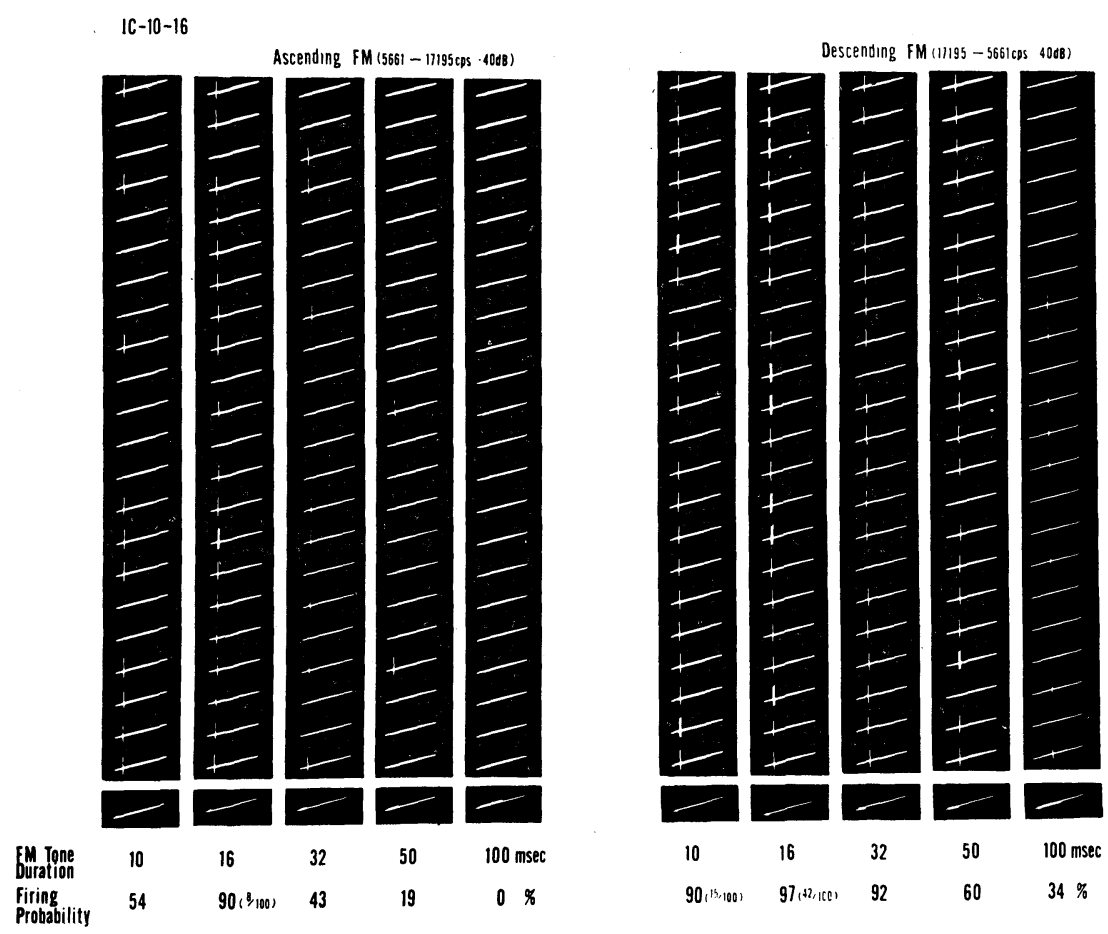

Fig. 2. A descending FM responding collicular neuron sensitive to a fast rate of modulation. 22 successive records are presented showing ascending and descending FM series at five different rates of modulation. The firing probabilities of each series are shown at the bottom. The bracketed values indicate the number of double firing responses to 100 stimuli. Note: The second column from the left in the descending FM series showing the highest responsiveness has the greatest firing probability and also the highest incidence of double firing of the neuron.

ing FM tones (right records) at five different rates of modulation are shown together with their respective firing probabilities. In this figure, the neuron responded mostly to descending FM tone, and was more responsive to a rather fast rate of modulation.

In contrast to these units (Figs. 1 and 2), Fig. 3 represents a neuron responding to descending FM tones that was more sensitive to a slower rate of modulation. This unit has a characteristic frequency of $4.0 \mathrm{Kc}$ with a moderate rate of spontaneous discharge. In 

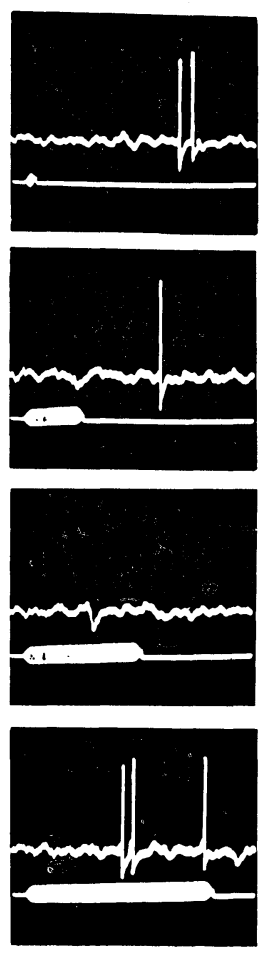

$$
2.6-8.0 \mathrm{Kc}
$$

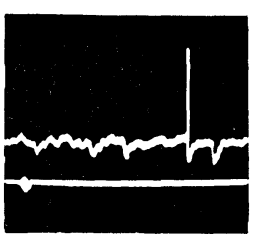

Threshold

( dB)

$-10$

$-30$

$-38$

$-50$

Fig. 3. A descending FM responding collicular neuron sensitive to a slow rate of modulation. Left and right columns represent ascending and descending FM series, respectively, at four different rates of modulation. Responses elicited by descending FM tones are shown in the 2 nd, $3 r d$, and last records. Otherwise, the spikes represent background activity. The characteristic frequency of this neuron was $4.0 \mathrm{Kc}$ and the modulating frequency range was between 2. $6 \mathrm{Kc}$ and $8.0 \mathrm{Kc}$. The number shown to the right of each sample record in the descending FM series represent the threshold of response to an individual stimulus. Note: Descending FM tones and slower rates of modulation lower the threshold of response.

this figure, unit responses were elicited by a descending FM tone, as shown in the second, third and bottom figures. The number of spikes increased with decreasing rates of modulation, and this was associated with a lowering of the response threshold of the neuron. Therefore, this particular unit had a descending directionality with a slow rate of frequency modulation. So far, no neurons at this level have 
yet been found to exhibit ascending directionality with a slow rate of modulation. It is possible that this type of neuron exists at higher auditory regions.

By analyzing the frequency components of the cat's voice (Fig. 4A), a sonagram was obtained (Fig. 4B). In B, the abscissa
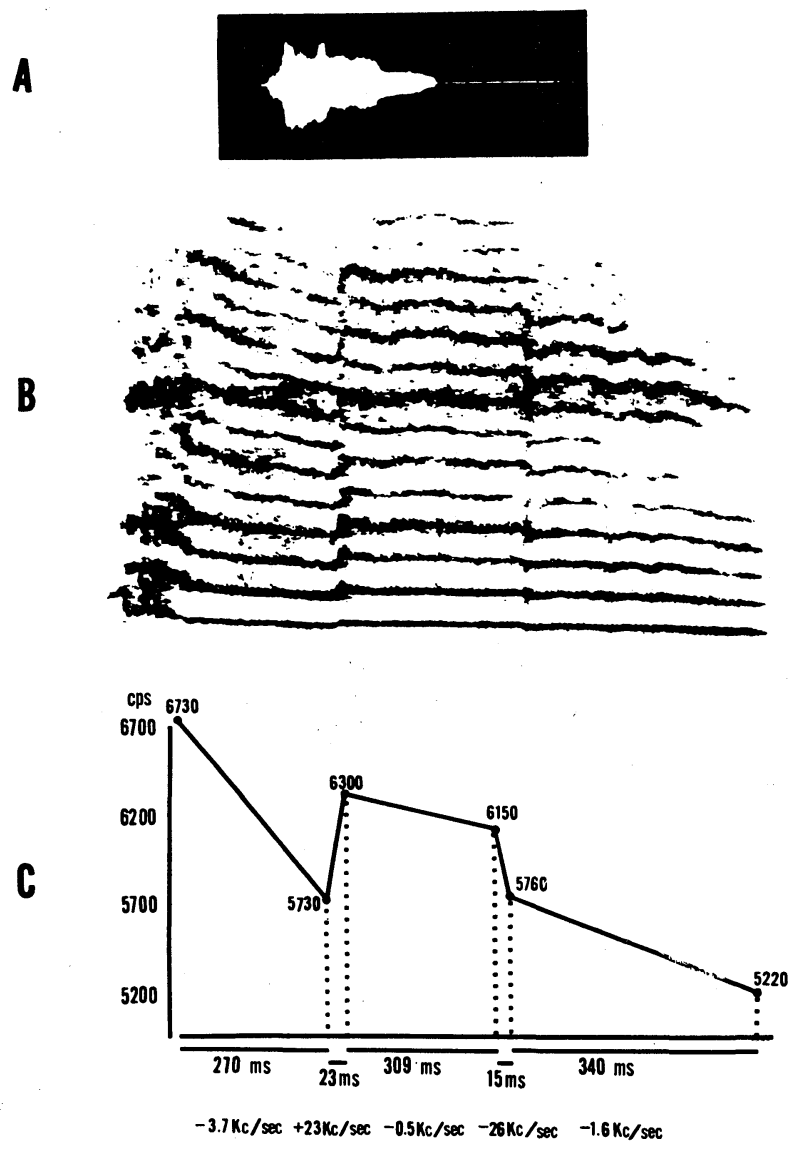

Fig. 4. The cat's voice (A) and its sonagram (B) showing frequency vs. time. The 11th harmonic of the sonagram (B) is traced schematically and the principal frequencies changing with time are indicated (C). The rates of modulation in the individual FM phases are expressed at the bottom as frequency-bandwidth per second. In these values, minus $(-)$ denotes the descending phase and plus $(+)$ the ascending.

represents the time and the ordinate indicates the frequency. There are many harmonics in the voice pattern, arranged almost parallel to each other over the same time course. Similar sonagram patterns were observed in several cats. The bottom trace of the sonagram 
shows the fundamental frequency with time. One of the prominent components was the 11th harmonic, approximate frequency changes with time of which are shown in Fig. 4C. Several FM components, either ascending or descending, and also different rates of modulation are shown in C. The modulation rate of this 11th harmonic was calculated and is presented at the bottom of Fig. 4C. Minus (-) indicates the descending FM and plus $(+)$ the ascending. Therefore, the neurons investigated in this report are characterized by their directional sensitivity to ascending or descending FM sound, and also to different rates of frequency modulation. The neurons are also characterized by their respective threshold frequencies.

It seems logical that these FM sensitive auditory neurons may respond to the particular FM components present in the cat's voice. These neurons may play an important role for analyzing the complex sound.

Similar studies in this area of research are progressing at the level of the medial geniculate and the auditory cortex.

Acknowledgement. The authors take this opportunity of thanking Prof. Y. Katsuki for facilities to do this work. Our thanks are also due to Dr. Lester Y. Ichinose, who kindly read the manuscript.

\section{References}

1) N. Suga: J. Physiol., 175, 50-80 (1964).

2) —-: J. Physiol., 179, 26-53 (1965); 181, 671-700 (1965).

3) I. C. Whitfield and E. F. Evans: J. Neurophysiol., 28, 655-672 (1965).

4) P. G. Nelson, S. D. Erulkar, and J. S. Bryan: J. Neurophysiol., 29, 834-860 (1966).

5) N. Suga: J. Physiol., 195, 51-80 (1968). 